\title{
The polymeric materials turning after workpiece processing with surface-active substances
}

\author{
Oleg Erenkov ${ }^{1, *}$, Daniil Yavorskii ${ }^{1}$ and Elena Yavorskaya $^{1}$ \\ ${ }^{1}$ Pacific National University, 680035 Khabarovsk, Russia
}

\begin{abstract}
The results of experimental investigations of surface quality after the lathe machining of polymeric materials subjected to preliminary treatment with surface-active substances are presented. The new combined method for polymer parts was developed. This method is based on directed change in the polymer properties by preliminary chemical treatment of the surface layer so as to reduce its resistance in the cutting zone. It has been demonstrated in experiments that the preliminary treatment of workpiece with surfactants ensures an improvement in quality of the surface machined by turning. This is indicated by a reduction in the roughness parameters by a factor of $2-4.5$, and the minimum roughness is achieved at a cut depth equal to $1.0-1.15$ the surfactant penetration depth into the material
\end{abstract}

\section{Introduction}

At present the combined methods are widely used in technology for the machining of structural materials. The technical essence of known methods is the impact on the surface of the workpiece with energy sources or an aggressive environment that enters into chemical interaction with the workpiece material [1,2]. The combined methods significantly increase the tools life and increase productivity and processing quality in comparison with the individual components of their methods.

On the basis of experimental data $[3,4,5]$, the new combined method for polymer parts was developed [6]. This method is based on directed change in the polymer properties by preliminary chemical treatment of the surface layer so as to reduce its resistance in the cutting zone.

The preliminary treatment produces a structure in which some of the chemical and intermolecular bonds are ruptured and the remainders are placed under stress. Thus, there is local embrittlement of a portion of the surface. The part is then subjected to turning. In that case, the chip formation is considerable changed: the removal of cut material is primarily due to brittle failure, since the deformation forces are reduced by the embrittlement. As we know, the energy of brittle failure is considerable less than the energy required for plastic failure $[4,5]$. Various defects in the vicinity of the leading deformations are formed at considerably lower stress, which promotes the nucleation of plastic shear and more stable propagation of major cracks ahead of the cutting edge along the line of action.

\footnotetext{
* Corresponding author: erenkov@list.ru
} 
The purpose of this work is an experimental study the quality of polymer materials turning after their preliminary processing.

\section{Experimental techniques}

The investigation was conducted on Caprolon and Textolite. The given materials belong to fundamentally different groups of polymeric materials - thermoplastics and thermosetting plastics, parts manufactured from which are widely used in different sectors of industry.

Lathe machining of experimental specimens was conducted on a model 16K20F3S47 universal screw cutting lathe. The geometric parameters and the material of the cutting tool and also the values of the cutting rate and feed rate were adopted on the basis of previously conducted investigations [2] and were kept constant throughout the present series of experiments. The quality of the turned surface was assessed from the values of the geometric parameters of roughness, the system of notation of which and the units for their monitoring were presented in [2].

The selection of the surfactants for the treatment of the polymeric materials was based on recommendations [7] made with account taken of the solubility of different polymers in corrosive media, namely: specimens of Caprolon were treated in acetone, and specimens of Textolite in toluene. The duration of preliminary treatment of workpiece with surfactants to ensure the required depth of penetration $t_{\mathrm{c}}$ of the medium was calculated by a well-known procedure [7, 8]. The results of this calculation are presented in Figure 1. Thus, to ensure the calculated depth of penetration of the surfactant $t_{\mathrm{p}}=2 \mathrm{~mm}$, the duration of treatment amounted to 4 and 7 hours for workpiece of Caprolon and Textolite respectively.

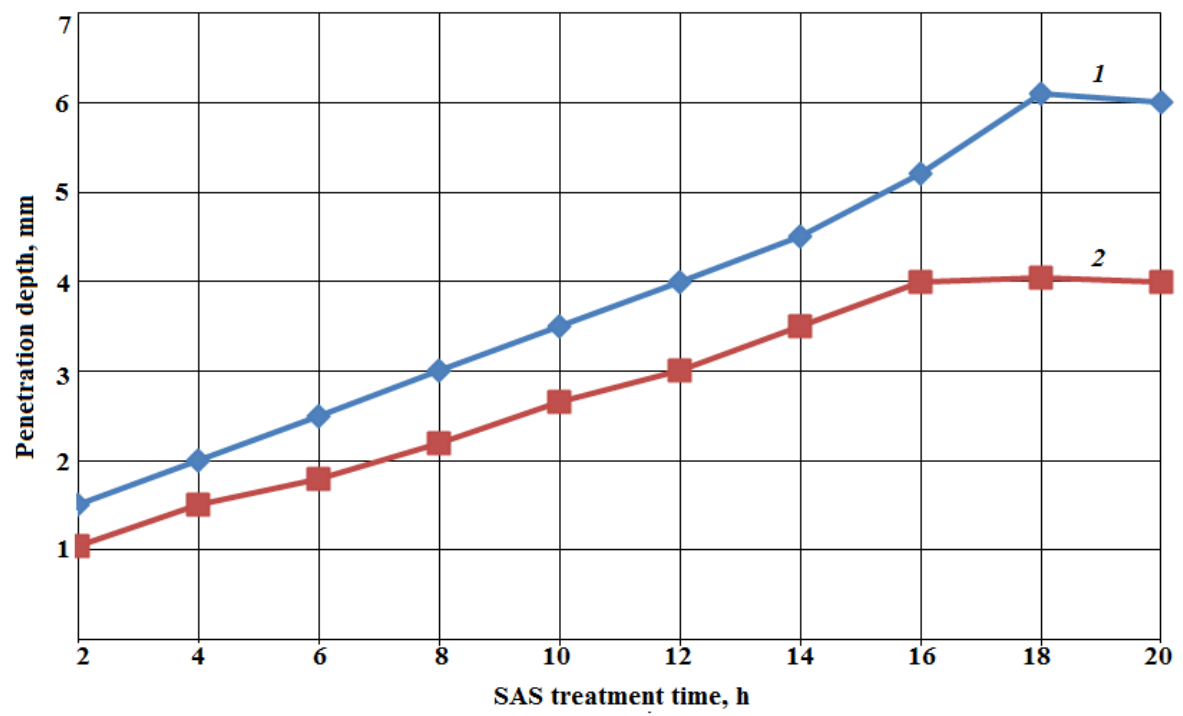

Fig. 1. The depth of SAS penetration: 1 - into Caprolon; 2 - into Textolite

\section{Results and discussion}

Figures 2 and 3 present profilograms of turned surfaces of parts manufactured from Caprolon and Textolite respectively. The depth of cut in the given series of experiments amounted to $2 \mathrm{~mm}$. Visual inspection of the profilograms makes it possible to conclude that the preliminary surfactant treatment of workpiece of Caprolon and Textolite promotes the 
formation of a higher- quality turned surface. A more detailed assessment of the roughness of machined surfaces was carried out on the basis of values of the combination of geometric parameters [2] given in Table 1.

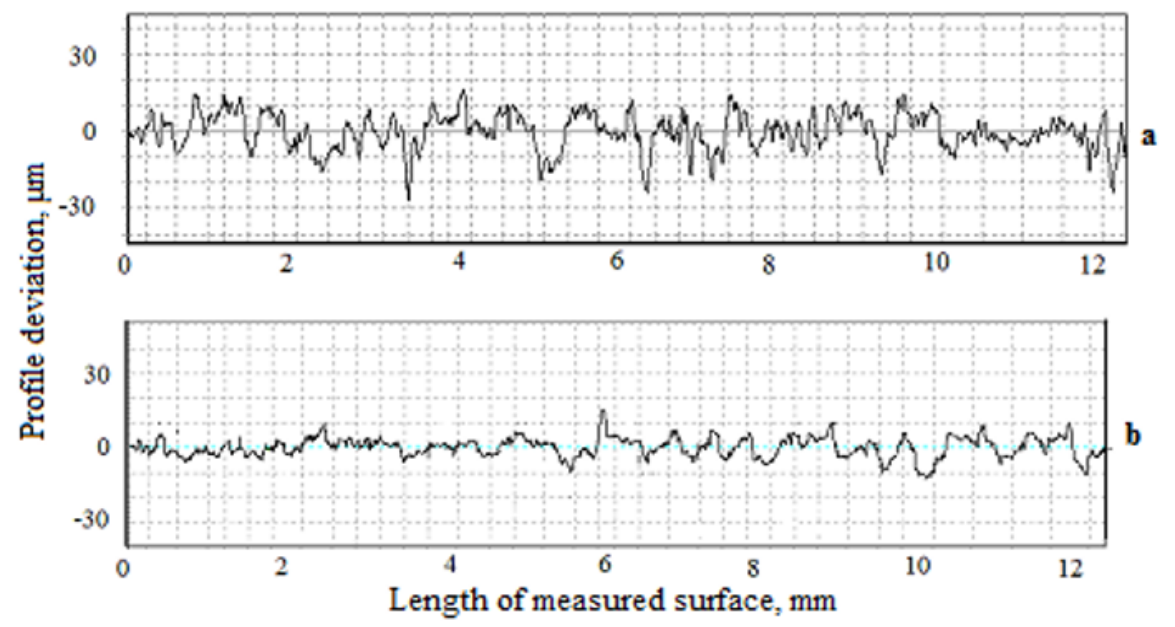

Fig. 2. Profilograms of the parts surfaces manufactured from Caprolon: $a$ - turning; $b$ - preliminary SAS treatment and turning.

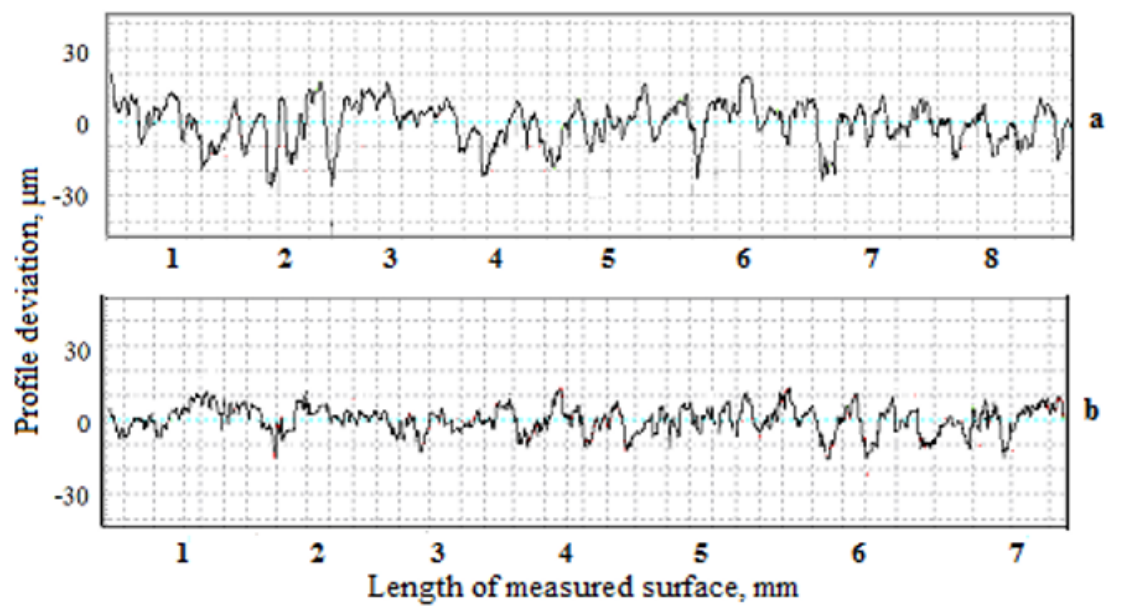

Fig. 3. Profilograms of the parts surfaces manufactured from Textolite: $a$ - turning; $b$-preliminary SAS treatment and turning.

An analysis of the roughness parameters (Table 1) confirms the fact established above that the preliminary treatment of workpiece manufactured from the investigated polymeric materials with the selected surfactants ensures an improvement in quality of the turned surface of workpiece. This is indicated by a reduction in the values of the roughness parameters from 1.8 to 4.5 for all the materials investigated.

During the lathe machining of polymeric materials, the depth of cut has a significant influence on the quality of the machined surface. In this connection, investigations of the roughness of the machined surface of Caprolon and Textolite as a function of the depth of cut were carried out. The level of roughness was monitored from the values of the arithmetic mean deviation of the profile $R$ a. The depth of cut $t_{\mathrm{c}}$ was varied from 1.5 to 2.6 $\mathrm{mm}$. 
Table 1. The results of the surface roughness investigation (depth of cut $t_{\mathrm{c}}=2 \mathrm{~mm}$ ).

\begin{tabular}{|l|c|c|c|c|c|c|c|c|}
\hline Material & Type of & \multicolumn{7}{|c|}{ Roughness parameters, $\mu \mathrm{m}$} \\
\cline { 3 - 9 } & treatment & $R_{a}$ & $R_{z}$ & $R_{\max }$ & $R_{p}$ & $R_{m}$ & $S_{m}$ & $S_{k}$ \\
\hline \multirow{3}{*}{ Caprolon } & $\begin{array}{c}\text { SAS } \\
\text { treatment and } \\
\text { turning }\end{array}$ & 4,39 & 11,6 & 15,2 & 7,6 & 5,89 & 0,88 & $-0,17$ \\
\cline { 2 - 9 } Textolite & $\begin{array}{c}\text { Turning } \\
\text { treatment and } \\
\text { turning }\end{array}$ & 7,6 & 22,72 & 35,97 & 14,02 & 21,95 & 0,33 & 0,27 \\
\cline { 2 - 10 } & Turning & 10,0 & 40,53 & 48,43 & 26,27 & 22,16 & 0,21 & 0,56 \\
\hline
\end{tabular}

An analysis of the graphic dependences (Figure 4) indicates that the distribution of parameter $R_{\mathrm{a}}$ of the machined surface of Caprolon (Figure 4, line 1) and Textolite (Figure 4 , line 2) is fairly non-uniformly.

At a depth of cut $t_{\mathrm{c}}<t_{\mathrm{p}}=2 \mathrm{~mm}$, the roughness of the machined surface is fairly high, higher than the roughness of the initial specimen. This is due to the embrittlement of the surface layer of the workpiece t material, i.e. the presence of a large number of external and internal defects in the form of cracks and microcracks formed owing to the surfactant treatment. The minimum roughness is achieved, other conditions being equal, at a depth of cut $t_{\mathrm{c}}=1.0-1.15$ tp.

This can be explained in the following way. An important feature of crystalline polymers is the fact that they consist not only of crystalline but also of amorphous regions. Here, the idea of the structure of the crystalline polymer as a combination of distinctly separate phases is an ideal; between the crystalline and amorphous regions there are areas with an intermediate degree of ordering, i.e. there is no distinct boundary between the crystallites and the amorphous regions, as one macromolecule can lie in several crystallites, passing through several amorphous regions $[9,10]$.

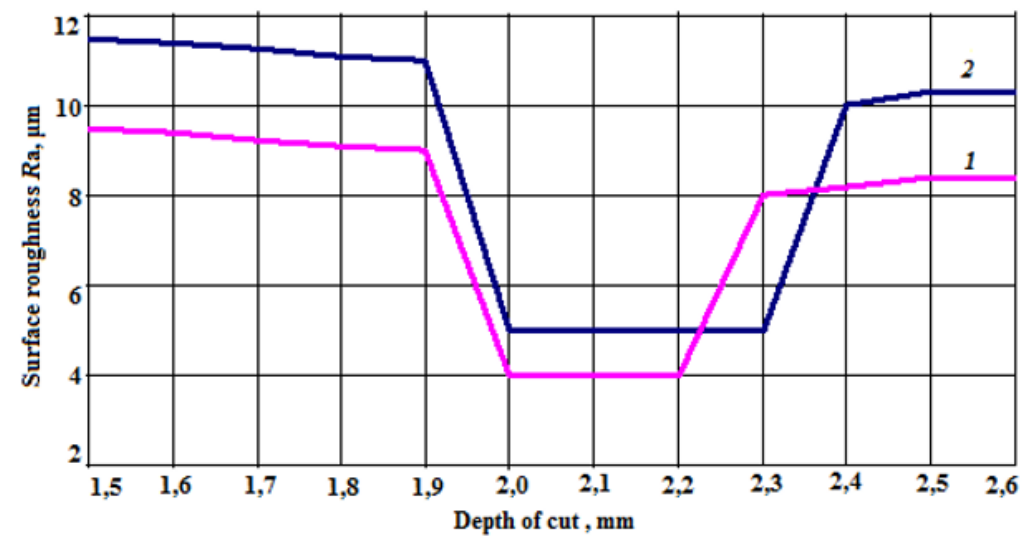

Fig. 4. The influence of the cut depth on the machined surface roughness: 1 - Caprolon; 2 - Textolite.

In this connection, on the basis of the above, it is possible, with a fair degree of confidence, to assert that a distinct boundary between parts of the material treated and not treated with surfactant does not exist, but rather that there is a transition layer extending to a 
depth slightly exceeding the depth of penetration tp. Owing to the effect of the surfactant, the transition layer has a material structure in which some of the chemical and intermolecular bonds are ruptured, while the others are weakened. On account of this, during subsequent turning, the process of deformation of the layer being cut is made easier, the work needed for the formation of new surfaces is reduced, and the resistance to cutting is reduced. All this ensures a more stable distribution of the main crack ahead of the cutting edge along the line of cut, and consequently a reduction in the level of roughness of the machined surface.

With further increase in the depth of cut $t_{\mathrm{c}}$, the roughness increases sharply to the level of roughness of a surface machined by turning without the use of a surfactant and remains practically constant.

\section{Conclusions}

On the basis of experimental data [3,4,5], the new combined method for polymer parts was developed [6]. This method is based on directed change in the polymer properties by preliminary chemical treatment of the surface layer so as to reduce its resistance in the cutting zone.

The presence of a surface layer of constant depth and embrittlement permits improvement in quality of the cut surface, since it ensures stable propagation of the major crack ahead of the tool's cutting edge.

It has been demonstrated in experiments that the preliminary surfactant treatment of workpiece ensures an improvement in the quality of roughness of the turned surface. This is indicated by a reduction in the roughness parameters from 2 to 4.5; here, the minimum roughness is achieved, other conditions being equal, at a depth of cut equal to 1.0-1.15 of the depth of penetration of surfactant into the material.

\section{References}

1. V.N. Poduraev, The Machining of Difficult-to-Machine Materials (Engineering, Moscow, 1974)

2. O. Yu. Erenkov, Combined Methods for Turning Polymer Composite Materials (Pacific National University, Khabarovsk, 2015)

3. V.N. Manin, A.N. Gromov, Physicochemical Stability of Polymers in Operational Conditions) Chemistry, Leningrad,1980)

4. A.A Tager, Physical Chemistry of Polymers (Chemistry, Moscow, 1978)

5. G.V. Bartenev, Yu.S. Zuev, Strength and Disintegration of Highly Elastic Materials (Chemistry, Moscow, 1994)

6. Erenkov O.Yu., Russian Patent 2494865, (51) MPK B 29 C 37/00. The method for treating plastic workpiece. No. 2012139273, appl. 12.09.2012, publ. 10.10.2013

7. Yu.S. Zuev, The Fracture of Polymers Exposed to Corrosive Media (Chemistry, Moscow, 1972)

8. O.Yu. Erenkov, Calculating diffusion parameters for a corrosive medium acting on polymer materials, Chem. Petr. Eng., v.45(7-8), pp.505-512 (2009)

9. E.M. Kartashov., The Structural Statistical Kinetics of Failure of Polymers (Chemistry, Moscow, 2002)

10. A.A. Askadskii, Yu.I. Matveev, Chemical Structure and Physical Properties of Polymers (Chemistry, Moscow, 1983) 